\title{
Educar en Bioética: el rol del directivo en el proceso de enseñanza del profesional de las ciencias de la salud
}

\author{
Educate in Bioethics: the role of the manager in the teaching process of the professional health sciences \\ Constantino Domínguez Barrera ${ }^{1, a}$, Mirtha Cervera Vallejos ${ }^{2, b}$, Franco León Jiménez ${ }^{3, c}$, César Ñique \\ Carbajal $^{4, d, e}$
}

\section{RESUMEN}

En función de las necesidades de investigación en formación de recursos humanos poco abordado en nuestro medio, y partiendo de la experiencia de vida dedicados a la docencia universitaria por parte de los autores, pretendemos realizar un análisis y propuesta sobre las cualidades profesionales y personales que debe poseer un directivo u autoridad académica en el campo de las ciencias de la salud. Para llegar a desarrollar un cargo de directivo dentro de la vida universitaria proponemos que la persona debe contar con una trayectoria académica y profesional reconocida y secuencial, con sólidos conocimientos en la gestión de la universidad a través de la certificación de un entrenamiento o programa de postgrado, así como también el haber plasmado en su quehacer académico la metodología de la investigación científica evidenciado en la publicación de obras que son consultadas por sus pares y estudiantes, asimismo es imprescindible que el directivo académico sea un ejemplo a seguir, sea ético, es decir que muestre coherencia entre su vida profesional y personal. En resumen, proponemos que para ser elegido autoridad en nuestro sistema universitario no basta cumplir con los requisitos que nos propone la ley, sino que deberíamos observar estas cualidades que a manera de propuesta creemos que sería determinantes para el buen gobierno de nuestras escuelas y/o facultades de ciencias de la salud.

PALABRAS CLAVE: Gestor de Salud, ciencias de la salud, investigación, gobierno, ética. (Fuente: DeCS BIREME).

\section{SUMMARY}

Depending on the research needs in human resources training little addressed in our environment, and based on the experience of life dedicated to university teaching by the authors, we intend to carry out an analysis and proposal on the Professional and personal qualities that an executive or academic authority must possess in the field of health sciences. In order to develop an executive position within the university life we propose that the person must have an academic and professional trajectory recognized and sequential, with solid knowledge in the management of the university through the certification of a training or postgraduate program, as well as having reflected in his academic

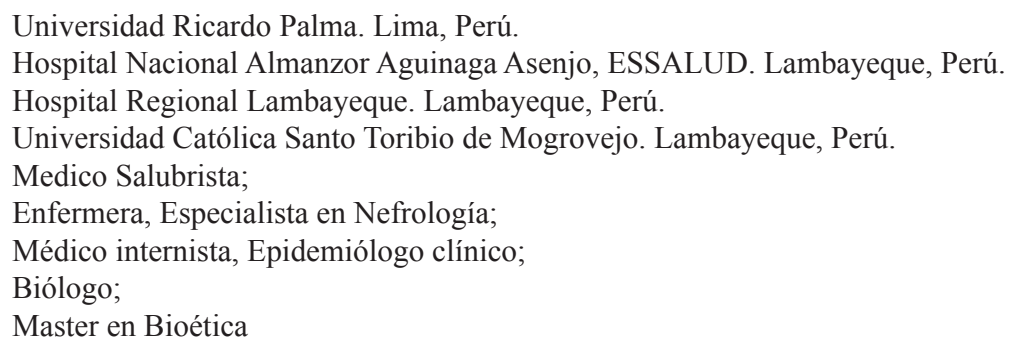


work the methodology of scientific research evidenced in the publication of works that are consulted by his peers and students, it is also imperative that the academic executive is an example to follow, be ethical, that is to show consistency between your professional and personal life. In summary we propose that to be elected authority in our university system is not enough to meet the requirements of the law, but we should observe these qualities that as a proposal we believe would be determinants for good governance of our schools and/or faculties of health sciences.

KEYWORDS: Health Manager, health sciences, research, government, ethics. (Source: MeSH NLM)

\section{INTRODUCCIÓN}

En el Perú una de las agendas temáticas nacionales en investigación, es el estudio de la formación de los recursos humanos en salud, tema planteado en su momento como producto de la actividad participativa y descentralizada a nivel nacional promovida por el Instituto Nacional de Salud reformulado para el periodo 2015 - 2021, a la fecha según un análisis bibliométrico de la producción científica en recursos humanos en nuestro país entre los años 2011-2014, solo se encontraron cuatro publicaciones en revistas científicas registradas en bases de datos como SCOPUS, LILACS y LIPECS de aproximadamente 882 publicaciones recuperadas (1).

Esto nos muestra la escasa mirada de los actores que participan en el complejo proceso de formación de los profesionales de las ciencias de la salud en cuanto a resolver problemas de uno de los temas prioritarios de investigación en el país, sumado a ello, la constante y creciente malapraxis de los profesionales de la ciencia de la salud es un hecho social, que merece ser también analizado con un riguroso criterio científico, este fenómeno considerado como una negligencia (o mala gestión por acción u omisión) como objeto de las normas jurídicas, nos parece que es una visión simplificada del problema para su control y prevención. Como sostiene el Principito de SaintExupéry: "lo esencial es invisible a los ojos" es decir, lo verdadero de las cosas no siempre es evidente y lo insignificante a veces es lo único que ponemos delante de nosotros (2).

Es frecuente observar que en nuestro mundo contemporáneo se nos hace difícil reconocer lo sustancial de la vida misma, fenómeno debido al progreso y desarrollo de la ciencia y tecnología, entendido en muchas ocasiones como fines que ayudan al "bienestar humano", sin embargo los resultados de dicha actividad nos han llevado a perder la característica fundamental del humanismo (capacidad para sentir afecto, comprensión o solidaridad hacia las demás personas), por el empoderamiento de la denominada "cultura de la deshumanización" de la acción humana. En consecuencia, bajo este enfoque la malapraxis es un código cultural susceptible de ser modificado por el proceso formativo $\mathrm{y} / \mathrm{o}$ educativo.

En este sentido las diversas profesiones de las ciencias de la salud que en su proceso formativo tienen como objeto o problema científico al ser humano, en su condición de unidad bio-psicosocio-trascendente, en permanente proceso de equilibrio inestable con su ambiente interno y externo, relacionado e interrelacionado a través de sistemas de comunicación biológica y social, deberán contemplar en esta perspectiva la estructura curricular, en la cual se integrarían las estructuras de las ciencias fácticas naturales y las fácticas sociales, como elemento fundamental en la formación de las competencias profesionales. En esta misma línea la incursión de asignaturas de corte humanista como la ética y la bioética en los planes curriculares de algunas profesiones de ciencias de la salud en el Perú tienen como finalidad transmitir valores éticos más apropiados, necesarios para los profesionales y sociedad en general, valores que deben formar parte constitutiva de quienes tienen la responsabilidad de formar profesionales, fundamentalmente de los docentes y autoridades académicas $(3,4)$.

Ante este panorama es fundamental hoy en día reflexionar sobre la educación en el nivel superior, específicamente sobre el rol del directivo o autoridad académica que participa de la noble tarea de formar profesionales, en el contexto filosófico, antropológico, ético y de responsabilidad social en la que se desenvuelve el proceso de enseñanza-aprendizaje. Este análisis se justifica sobre la base de una mirada humanística y ética que persigue desarrollar estos elementos como competencia de los profesionales de las ciencias de la salud.

Por lo expuesto pretendemos desarrollar la siguiente cuestión de fondo: ¿Qué cualidades personales y acciones específicas debería desarrollar o poseer el 
directivo o autoridad académica, que participa en el proceso formativo de los profesionales de las ciencias de la salud, en el Perú?

Para llegar a comprender y entender la misión que tiene todo profesional de la academia en su estatus de directivo o autoridad, debemos asumir un análisis interno muy concreto sobre la persona, ya Confucio decía: "uno que no sepa gobernarse a sí mismo, como sabrá gobernar a los demás", así también será necesario especificar ciertas habilidades directivas y diseño de acciones inherentes con su labor, de ahí la relevancia de la ética y de la bioética como disciplinas que rigen o ayudan a discernir el comportamiento humano en el campo de la ciencias de la salud, todo este análisis quedaría vacío sin vislumbrar el impacto de todas estas acciones en lo social, en la medida que se incorpore una mirada altruista y transcendente de la actividad de gobernar para los demás.

\section{Rol del directivo o autoridad, en el proceso formativo de los profesionales de las ciencias de la salud.}

Dirigir una institución de educación superior amerita reflexionar sobre ciertas cualidades y circunstancias propias de la vida universitaria, en primer lugar, consideramos que el enfoque de dirigir tiene que estar centrado en el servicio que brinda este tipo de instituciones, cuya finalidad es la formación de profesionales, sin embargo, existen otros aspectos a tener en cuenta en nuestro análisis porque están relacionados entre sí, los mismos que ayudarían a conseguir el objetivo final. Por esta razón un elemento sustancial es la del directivo o autoridad académica, quien está llamado a ser un ejemplo vivo de la actividad propia de la sindéresis, este hábito según lo analiza Leonardo Polo cumple la función de actualizar las potencias de la inteligencia y la voluntad, por ello cualquier directivo acierta, si ayuda a que las energías de los trabajadores que tiene bajo su cargo se pongan en marcha, porque contribuye a motivar su inteligencia y voluntad y a través de ellas el resto de potencias sensibles, mediante una actitud atenta, cercana y vigilante, respetando su libertad. Aspecto que requiere por parte del directivo en primer lugar que aprenda a pensar y enseñe a pensar al personal, que no es otra cosa que saber argumentar las propias opiniones, especialmente en los docentes universitarios de las ciencias de la salud, cuyo objeto de su actividad es la centralidad, seguridad y protección de la vida humana (5).
En este sentido, Carlos Llano (6) manifiesta que existen tres tipos de actividades directivas que promueven una acertado gobierno de personas, las mismas que pueden trasladarse al escenario académico, estas estarían configuradas en los procesos de diagnóstico, decisión y mando, operaciones que involucran una serie de valores y virtudes que en el momento de interactuar tendrán un impacto tanto en el exterior como el interior de la propia persona que los desarrolla como de los que reciben la dirección, para el caso del diagnóstico de la situación que le toca dilucidar al directivo es importante que ponga en práctica la objetividad y la humildad, en el tratamiento de la decisión le merece la operatividad de dos virtudes tales como la magnanimidad (con su correspondiente afán de logro) y la audacia (con su correlativa capacidad de riesgo) y en relación al mando propiamente, es necesario ejercer cinco cualidades: el autodominio, la constancia en el esfuerzo, la fortaleza ante las dificultades, la confianza en los demás y la lealtad.

Gobernar personas representa lo más valioso en cualquier sistema de la sociedad, observamos la importancia de la formación integral para que podamos vivir el sentido subjetivo de nuestro trabajo y lo trascendental del mismo, sobre todo debemos considerar que el fin de nuestras acciones como líderes será siempre buscar el bien de los demás, en este sentido las dimensiones del hombre que ejerce gobierno deberían contextualizarse y analizarse cada una a detalle, con la finalidad de vislumbrar un gobernar bien, la misma que no es una tarea fácil ni rápida, toma tiempo, dedicación y sacrificio. Un directivo preocupado por su ser, asume el compromiso de auto conocerse, lo cual cohabita como una tarea difícil, requiere de cierto esfuerzo, empeño, capacidad crítica, de observación y de análisis, estas características son propias de la persona reflexiva, emergen cuando se está atento asimismo en relación con los demás, denominado por Leonardo Polo (5) como intersubjetividad que devela como se es en ese momento y el querer conocerse denota una acción libre, por tanto una acción ética, así resulta atrayente conocer los defectos para mejorarlos y en ese esfuerzo logran autenticidad, ya que ante los errores se rectifica, de tal manera que no sólo existe un interés individual sino con actitudes de apertura, de reconocimiento del otro, trabajo en equipo para la solución de problemas sin discriminaciones de edad, sexo, religión, situación económica, etc., buscando la coherencia de vida, las virtudes de la convivencia y el respeto a la diversidad cultural. 
Y como el ser preside al hacer, y el ser se crea en relación con otros, el directivo entonces se transforma en modelo para sus gobernados, porque la genuina autoridad, en cualquier campo de la vida humana, no es imposición despótica, sino prestigio moral que depende del ejemplo, surge del prestigio ético que uno tenga y de su ejemplaridad en la conducta, el "ordeno y mando" cae en el peor de los desprestigios cuando la conducta del que manda entra en la incoherencia (7).

Por esta razón se necesita aprender a aprender constantemente, y el directivo no puede retraerse de tremenda responsabilidad puesto que mejorará en la medida en que sus actos sean buenos, poniendo los medios para lograrlo de tal forma que garantice en los demás la imaginación, la creatividad, la innovación, con el uso adecuado de sus capacidades, aptos para gobernar sus acciones y la propia vida con libertad responsable que garanticen el desarrollo de la cultura y las sociedades. Mejoras en el hacer que reclama del directivo movimiento, acercamiento, trato, una vida activa ya que manejar información y sobre todo a las personas requiere acciones específicas y concretas. Los buenos directores son reflexivos; saben cómo aprender de su propia experiencia, exploran numerosas posibilidades y son capaces de rectificar y ejecutar una estrategia alternativa, si no funciona la que están empleando, procurando unidad y síntesis para decidir, mejoras que lo posesiona, también en el buen uso de las tecnologías existentes, las cuales no deben convertirse en símbolo de deshumanización sino por el contrario, estar sujetas al servicio del hombre y su bienestar (8).

Luego, para tener es necesario haber trabajado y cultivado el propio ser y hacer en el logro del propio perfeccionamiento, el tener es por añadidura, consecuencia de actos buenos, de virtudes vividas con heroísmo y alegría, dominar los defectos y optar por tener planes llenos de vida, como por ejemplo ser más eficaz en el trabajo directivo y/o docente, el cual le puede llevar a perfeccionar, asumiendo el querer los deberes propios con gran responsabilidad que inducen a omitir la pereza y dedicar tiempo y ganas a formar, ya que el trabajo de formar y educar requiere disciplina, método, constancia, resistencia, más que física espiritual, así se forma para lograr personas que saben dominarse a sí mismos, sin inestabilidad en las tareas y sin vacío prácticos que dejan a medio hacer las labores, y logren no sólo el éxito, el cual también repercuten en la familia y sociedad, sino también la retribución económica por el trabajo bien hecho con conciencia del deber que es abnegación que sin lugar a dudas proporciona felicidad, una vida lograda.
Por el contrario, explican Scalzo y García-Alvarez (8), un director que no se esfuerce por ser buena persona, fracasa como director, si un director miente para obtener un beneficio, puede que técnicamente alcance sus objetivos, pero prácticamente empeora. Así, el reconocimiento de la naturaleza propia de la dirección implica la aceptación de la superioridad de la teoría normativa para una comprensión más realista de la vida de las organizaciones y su contribución al desarrollo humano y social.

\section{La ética en el proceso de gobernar o dirigir}

El término ética se deriva del vocablo griego ethos, que significa morada o lugar. En la actualidad, la ética se define como la disciplina filosófica que estudia el comportamiento moral del hombre en sociedad. Es, por tanto, una rama de la filosofía, cuyo objeto de estudio es la moral, la cual es inherente al hombre como ser social. A partir de esta relación entre la ciencia y su contenido se comprende que la ética es la teoría de la moral, la que puede definirse como un sistema de opiniones, representaciones, normas y evaluaciones sobre la regulación de la conducta de los individuos que viven en sociedad y ante una situación concreta (9).

Sólo en la medida en que el directivo viva la ética desde su ser personal, desde si mismo podrá promover gozosa y eficazmente la persona de quienes tiene bajo su responsabilidad. Esto significa que todo directivo debido a su ser, se hace responsable, crece al entregar lo que es, puede prosperar y llegar a la excelencia. Toma el ejercicio de gobernar como vocación y como modo de vida, se compromete a extraer de sí mismo toda su riqueza, expresada en su actuar ético; $\mathrm{y}$, cuando se evidencia una actuación ética, da como consecuencia que sea modelo de actuación personal y profesional.

Realidad que es corroborada por Pastor L (10), al afirmar "Quien es incapaz de respetar la dignidad humana se convierte también en un depredador del mundo circundante. Quien sólo busca su utilidad, sea en bienes deleitables o económicos, no se detendrá ante un ecosistema, que siempre será para él una propiedad, un dominio, sujeto a su razón instrumental".

Entonces quien no es ético, como afirman Polo y Llano (11), vive sumido en la mentira y suscitarán miedo a quienes les rodean especialmente a los más próximos y el miedo y la mentira "rompen la vida social, aíslan a los seres humanos, hacen imposible la 
confianza y si la mentira se arraiga en uno mismo, no se dirá nunca la verdad y nunca se confiará en nadie".

En consecuencia el directivo ético sabe dirigir y gobernar, aplica su propia humanidad, para lograr el cambio de conducta de en los demás, cambio logrado porque ellos mismo lo desean al percibir que el gestor los motiva, da ejemplo, les comunica el objetivo común que los conducirá al logro de las metas propuestas y sobre todo porque actualiza sus potencialidades a base de innovaciones originales en su enseñanza, de allí que la función de la ética lleve consigo formar actitudes éticas de buscar el bien, integrar de forma sistémica la enseñanza porque la persona es sistémica, adquirir compromisos con la propia formación y de las personas que rodean al estudiante constituyéndose en un elemento dinamizante de la identidad propia, lo cual da prestigio, y posicionamiento.

\section{La investigación científica y el directivo}

Uno de los pilares de la actividad universitaria, es la producción de conocimiento científico socialmente validado capaz de generar soluciones creativas en las múltiples áreas del quehacer social, las instituciones académicas de nivel superior viven el día a día de la gestión de la investigación patentando en diversos procesos como la planificación estratégica, la evaluación del desempeño organizacional, la evaluación de los sistemas de movilización de recursos, la evaluación de los procesos gerenciales, el desarrollo organizacional, la evaluación de la cultura organizacional y el modelo estructura-funcional. Gestionar una Universidad significaría en cierta medida gestionar en investigación, ya que, a través del método científico, este nos brinda herramientas y recursos en la debida planificación de las actividades de gobierno donde se obtienen, procesan y analizan información pertinente tanto interna como externa, con la finalidad de evaluar la situación presente, así como su nivel de competitividad con el propósito de anticipar y decidir sobre el direccionamiento de la Universidad (12).

La Universidad centrada en la investigación, en la innovación, en la internacionalización y en la excelencia, buscará evaluar constantemente la docencia de calidad, la innovación/investigación pedagógica, la investigación y al propio docente investigador, ya que este último elemento mediante la investigación, puede continuar creando conocimiento científico y mejorar, de este modo, su campo científico; pero también de la investigación pedagógica que le reportará nuevas propuestas metodológicas adaptadas a sus alumnos y materias, para innovar en su realidad y en su contexto docente. Para conseguir estos objetivos será necesario mantener una actitud de constante reflexión y crítica, de auto perfeccionamiento, de formación, de compromiso ético con la profesión, entre otros elementos, evidenciándose que una debida preparación y categorización del docente universitario en esta perspectiva, sería la mejor propuesta para poder contar con la promoción de ser gestores o directivos académicos de la educación superior (13).

En correlato a lo planteado en el párrafo anterior se encontró un trabajo de investigación donde se encuestaron de manera anónima a 491 profesores universitarios de 41 facultades de medicina en los Estados Unidos de Norteamérica, sobre las características de liderazgo que debería poseer un decano, máxima autoridad académica de una facultad, en cuyos resultados se pudo observar que para la gobernanza este deberá poseer una área de especialización en visión estratégica, capacitación o entrenamiento que debe ser formal o acreditada, esta cualidad podría recomendarse sobre la base de haberse formado como docente investigador en su carrera docente universitaria con perspectivas de ayudar al gobierno de la universidad, resaltando ciertas cualidades a desarrollar tales como el deber de explicar sus decisiones, comunicar abiertamente temas difíciles e incomodos, tener la capacidad para admitir la confianza promovida por error en lugar de ser vista como debilidad; así mismo como valor organizacional a promover, el más solicitado por parte de los docentes encuestados hacia sus autoridades, es la de ser integro, es decir guardar la coherencia entre lo que se dice y lo que se hace, ya que esto genera confianza y es parte de la ética organizacional (14).

\section{Impacto de gobernar con ética en la sociedad}

Hoy en día no existe institución pública o privada en el ámbito de la vida en sociedad que no se encuentre inmune de hechos de corrupción, actos que ponen en evidencia los antivalores como la codicia, avaricia y anhelo de poder, elementos reñidos con la vocación de la persona que es buscar hacer el bien a los demás, esta situación se vive porque se ha perdido de vista que el vivir va ligado a nuestra ética, ya que hacerlo de esta manera permitiría al individuo tener una conducta libre y responsable orientada a la realización del bien mediante el cumplimiento del deber. La ética muestra los distintos comportamientos existentes en el ser humano. Una vez conocidos estos, es posible 
formarse un buen carácter. Cuando un individuo posee ética se despierta en él un espíritu de servicio $\mathrm{y}$, en consecuencia, actúa con responsabilidad en el ejercicio de sus funciones, no ofendiendo, ni abusando del poder que ejerce, no hurtando, ni engañando, no siendo soberbios, al contrario, ayudando, enseñando con sencillez, siendo solidarios, responsables con sus tareas y finalmente ofreciendo resultados (15).

Según Cortina (16), gobernar con ética genera entre otros aspectos: a) compromisos y confianza, haciendo que el mundo sea más económico en dolor evitable y también en dinero, de tal manera que los vacíos éticos que han servido para que prolifere la corrupción en diferentes ámbitos profesionales, no sigan incentivándose. b) Labrarse un buen carácter que cultive las virtudes y aleje los vicios, permitiéndonos ser felices y justos. c) Querer cuidar para combatir el individualismo egoísta y procurar la servicialidad, la comprensión inteligente y la amabilidad. d) Conquistar solidariamente la libertad, procurando recuperar la noción de un espacio en que cada ser humano sea entendido como fin en sí mismo, para construir una "sociedad sin dominación y, por lo tanto, una sociedad sin humillación. e) La felicidad humana con vida buena, implica saber reconocer y estimar lo que vale por sí mismo tal como el respeto por la dignidad de las personas, así como la compasión. Estos son sentimientos que fundamentan los Derechos Humanos y legitiman la exigencia de su cumplimiento en cualquier lugar del mundo.

\section{Propuesta: Cualidades del directivo universitario de las ciencias de la salud}

Por todo lo expuesto y analizado desde una mirada al interior de lo que significaría gobernar personas en el complejo proceso formativo de los profesionales de las ciencias de la salud en el contexto universitario peruano, a manera de propuesta sugerimos tener en cuenta los siguientes elementos para el performance de la persona en su condición de directivo o autoridad académica:

a) El directivo universitario debe conocer la vida universitaria y haber realizado carrera docente universitaria, ya que la esencia de su labor radica en su constitución académica-profesional, como una expresión relacional indivisible y univoca de la experticia en educación con la experticia en la carrera profesional de las ciencias de la salud de la que forma parte, esta dinámica la podrá trasladar en experiencias de aprendizaje ahora en la faceta de autoridad sobre las personas que ejerce gobierno. b) Deberá poseer formación en gestión de recursos humanos debidamente acreditada, dicha actividad consolidará las capacidades y cualidades que debe poseer el directivo académico para llegar a ser líder en la gobernanza institucional en la búsqueda no solo de solucionar los desafíos y las dificultades, sino también sobre la promoción del talento humano que tiene bajo su disposición, esto podría complementar la disposición sobre los derecho del docente contemplada en la ley universitaria peruana $\mathrm{N}^{\circ} 30220$, cuyo artículo 88.2 a la letra menciona: elegir y ser elegido en las instancias de dirección consulta según corresponda.

c) Consideramos también que una de las facetas que deberá desarrollar el directivo es la de ser investigador, porque la actividad científica en si misma ayuda a tener una mirada de la realidad abierta a muchas posibilidades donde forma parte la contemplación de los hechos que en ocasiones no pueden ser percibidas por el que no tiene esta práctica, asimismo el método científico te permite actuar con veracidad, autonomía, responsabilidad y prospectiva en este caso para el buen gobierno de la universidad-facultad, entre otros beneficios para la vida universitaria ya evidenciadas y que hoy en día son materia de acreditación y certificación de la calidad en el proceso de enseñanza-aprendizaje.

d) Ser una persona íntegra y coherente en su forma de pensar y hacer, con actitud abierta y reflexiva, característica que nos atrevemos a proponer como un directivo bifocal, es decir no solo comprometido con la institución o personas a las cuales se debe como autoridad sino y sobretodo preocupado por su crecimiento como persona, que a la luz de un análisis profundo de su actuar como profesional y personal, se pueda determinar un perfil de coherencia humana entre el ejercicio de las virtudes y valores, lo que le permitiría obtener excelencia, ser comprometido y ético, a pesar de la imperfección y de la naturaleza inacabada que posee toda persona.

\section{Conflictos de intereses:}

Los autores declaran no tener conflictos de intereses.

\section{Correspondencia:}

César Ñique Carbajal

Av. Miguel Grau 1552 La Victoria Chiclayo Perú

Teléfono: 51743151561

Correo electrónico: cnique@usat.edu.pe 


\section{REFERENCIAS BIBLIOGRÁFICAS}

1. Romani F, Roque J, Vásquez $T$, Mormontoy $H$, Vásquez $\mathrm{H}$. Análisis bibliométrico de la producción científica sobre las agendas nacionales de investigación en el Perú 2011-2014. Ann Fac med. 2016; 77(3): 241249. (Fecha de acceso 28 de mayo del 2018) Disponible en: http://www.scielo.org.pe/scielo.php?script=sci arttext\&pid $=$ S1025-55832016000300008\&lng=es

2. Domínguez-Barrera C, Ñique-Carbajal C. Crisis actitudinal en el estudiante de las ciencias de la salud. Rev cuerpo méd. HNAAA 2017; 10(3):175-77.

3. León F. Enseñar Bioética: Como transmitir conhecimentos, atitudes e valores. Acta bioeth. 2008; 14(1):11-18.

4. Cubas F, León F, Ñique C. Educar en Bioética: Retos para el profesor universitario de ciencias de la salud. Rev Med Hered. 2018; 29(1): 46-51. DOI: https://doi.org/10.20453/rmh.v29i1.3261

5. Polo L. Ayudar a crecer. Cuestiones Filosóficas de la Educación. Pamplona: Eunsa; 2006.

6. Llanos C. Caracterología del directivo al inicio del siglo XXI. Revista Empresa y Humanismo.2002; 5(2):321-344.

7. Huerta V. Autoridad y ejemplaridad. 2018. (Fecha de acceso 20 de junio del 2018) Disponible en: http://www.serpersona.info/2018/06/autoridad-yejemplaridad.html

8. Scalzo G, Garcia-Alvarez S. El Management como práctica: una aproximación a la naturaleza del trabajo directivo. Revista Empresa y Humanismo. 2018; 21(1):95-119. doi: http://dx.doi.org/10.15581/ 015.XXI.1.95-119.
9. Pernas M, Ortiz M, Menéndez A Consideraciones sobre la formación ética de los estudiantes de Ciencias Médicas. Educ Med Super. 2002; 16(2): 113-119. (Fecha de acceso 20 de junio del 2018) Disponible en: http://scielo.sld.cu/scielo.php?script=sci_arttext\& pid=S0864-21412002000200005\&lng=es.

10. Pastor L. El hombre como centro integrador de la Bioética Cuadernos de Bioética. 2016; 27(3): 285287. (Fecha de acceso 20 de junio del 2018) Disponible en: http://aebioetica.org/revistas/2016/27/91/285.pdf

11. Polo L, Llano C. Antropología de la Acción directiva. Madrid: Unión Editorial; 1997.

12. Royero, J. Gestión de sistemas de investigación universitaria en América Latina. Revista Iberoamericana De Educación. 2003; 33(1):1-19.

13. Mas-Torelló O. Las competencias investigadoras del profesor universitario: la percepción del propio protagonista, de los alumnos y de los expertos. Revista de Currículum y Formación de Profesorado. 2014; 18(3):255-273.

14. Hromas R, Leverence R, Mramba LK, et al. What a medical school chair wants from the dean. Journal of Healthcare Leadership. 2018; 10:33-44. DOI: https://doi.org/10.2147/JHL.S158937

15. Bautista D. Fundamentos de la ética en el servicio público. Ciudad de México: Instituto Nacional de Administración Pública; 2017. (Fecha de acceso:28 de agosto 2018) Disponible en: http://ri.uaemex.mx/ handle/20.500.11799/68827

16. Cortina A. ¿Para qué sirve la ética? Barcelona, España: Paidos; 2013.

Recibido: 28/08/2018

Aceptado: 25/09/2018 